\title{
Viable Extreme Preterm Birth and Some Neonatal Outcomes in Double Cerclage versus Traditional Cerclage: A Randomized Clinical Trial
}

\author{
Farzaneh Broumand, ${ }^{1,2}$ Fatemeh Bahadori, ${ }^{1}$ Tahereh Behrouzilak, ${ }^{1}$ \\ Zahra Yekta, ${ }^{3}$ and Farkhondeh Ashrafi ${ }^{1}$ \\ ${ }^{1}$ Obstetrics and Gynecology Department, Urmia University of Medical Sciences, \\ Urmia 57146-15463, Iran \\ ${ }^{2}$ Shahid Motahhari University Hospital, Kashani Street, Urmia 57146-15463, Iran \\ ${ }^{3}$ Department of Epidemiology, Faculty of Medicine, Urmia University of \\ Medical Sciences, Urmia 57146-15463, Iran
}

Received 23 June 2011; Revised 7 August 2011; Accepted 8 August 2011

Academic Editor: Hatim Omar

The pregnant women at higher risk of preterm labor, referred to the perinatal clinic of Kosar University Hospital in Urmia district of Iran, were enrolled into a parallel randomized clinical trial. In the investigational arm of the clinical trial, a double cervical cerclage procedure was performed addition to McDonald cerclage. In the control group however, only McDonald cerclage was performed. Extreme preterm labor ( $\mathrm{GA}<33$ weeks) was the primary endpoint of this clinical trial. Age, gestational age at cerclage time, and gravidity were not found to be statistically different between the groups. Means of gestational age were 37.4 and 36.2 weeks, respectively, for the investigational and control groups. The gestational age was 1.2 weeks longer for double cerclage group but the difference was not found to be statistically significant. Preterm birth before 33 weeks of gestation was not experienced by any of the patients who received double cerclage, but five women in control group developed such an extreme preterm labor $(P<0.05)$. The absolute risk reduction in using double cerclage over traditional method was 18 percent (95\% confidence interval, $4 \%-32 \%$ ). Double cerclage appeared to have higher efficacy than traditional cerclage in preventing preterm labor $<33$ weeks of gestation.

KEYWORDS: preterm birth, incompetent cervix, double cerclage, cervical cerclage, clinical trial 


\section{INTRODUCTION}

Preterm birth before 37 weeks of gestation is the most common catastrophic and costly complication of pregnancy. Preterm birth remains the leading cause of neonatal morbidity, mortality, and consequent sequels. Although preterm birth before 37 weeks of gestation is of high importance by itself, it can also be subcategorized based on gestational age. For example, "near-term birth" terminology is used for those preterm deliveries close to 37 th week of gestation. Researchers may also like to call those preterm births farther away from term delivery, as nonviable ( $<28$ weeks) extreme preterm birth and viable $(28<$ GA $<$ 33 weeks) extreme preterm birth. Regardless of trivial differences in terminology, it seems that viable but extreme preterm birth can be considered as a challenging situation for medical care providers. Interventions to prevent the condition will be of great value if proved to be efficient and safe.

A major cause of preterm labor, as an appropriate target for interventional studies, remains to be the incompetent cervix. Incompetent cervix is a clinical diagnosis with recurrent painless cervical dilatation and spontaneous loss of pregnancy products [1]. A history of cervical incompetence predisposes to repeated similar event in subsequent pregnancies [2]. Previous research was started to use cerclage methods on the basis of mechanical strengthening of incompetent cervix. Nevertheless, no strong evidence of the efficacy of traditional cerclage methods was available until recently [3-5].

Occult chorioamnionitis has long been regarded as a major cause of preterm birth. The most common pathway for intrauterine infection is the ascending route from vagina and cervix [6]. Increasing evidence suggests that cervical mucus plug plays more than just a mechanical role in protecting the fetoplacental unit against ascending infection from the vagina [7]. Although in many cases it is impossible to determine whether weakness of the cervix or ascending infection is the primary cause, application of a procedure to prevent both mechanisms seemed to be promising. Double cerclage can be considered to cover this need but there is a paucity of information from randomized clinical trials in different settings.

The aim of this study was to compare efficacy of double cervical cerclage (cervical occlusion suture plus traditional McDonald suture) with simple McDonald suture on lengthening the gestational age and preventing extreme viable preterm pregnancy.

\section{METHODS}

\subsection{Participants}

The study was conducted in the perinatal clinic of Kosar University Hospital in Urmia, Northwest Iran between October 2009 and February 2011. Study was conducted under supervision of the deputy for research in Urmia University of medical sciences. It was a randomized clinical trial conducted in parallel design with two equal wings to compare two surgical methods. The pregnant women at higher risk of preterm labor referred to the perinatal clinic of Kosar University Hospital were enrolled.

Women with singleton pregnancy 13-18 weeks of gestation who had a history of spontaneous preterm delivery or cervical shortening and cervical length $<25 \mathrm{~mm}$, detected by transvaginal ultrasonography, were eligible to be included in this study. The exclusion criteria were as follows: obstetrical complications like vaginal bleeding and premature rupture of membranes, major fetal anomalies, known uteral anomalies, and indications for emergency cerclage. Cervical length was measured using transvaginal ultrasonography. Gestational age was determined based on the last menstrual period (LMP) in case of regular menstrual history, otherwise by ultrasonography during the first trimester.

\subsection{Trial Arms}

The trial included two parallel arms. A total of 56 participants were enrolled into this study and were randomly assigned to be equally included in both arms of the study. Block randomization was used to ensure equal group sample size and increase the study power [8]. This sample size powered the study up 
to $70 \%$ at $90 \%$ confidence level to detect the observed $18 \%$ risk reduction for the primary outcome of the study. STATA version 11 (STATA corporation, College Station, Texas, USA) was used to do the power estimation. Hopefully, none of the participants were lost to followup, all 56 subjects continued with the study. In the investigational arm, double cervical cerclage was performed as a cervical occlusion suture plus McDonald cerclage. Cervical occlusion suture was performed with a purse string nylon 2-0 suture at the external os with $5 \mathrm{~mm}$ distance from edge and with $10 \mathrm{~mm}$ depth. In control group, McDonald cerclage was performed with a $5 \mathrm{~mm}$ tape white polyester suture produced by SUTULENE.

Single-dose prophylactic intravenous antibiotic was administered. After the operation patients were asked to take rest in bed for 24 hours, followed by one-day mobilization prior to discharge from the hospital. Postdischarge patient management was similar in both groups. They were recommended to restrict their physical activity and to abstain from sexual intercourse during pregnancy. Women were readmitted to hospital if vaginal bleeding or preterm rupture of membranes was noted. They followed with regular prenatal visits at prenatal clinic of the hospital. Cervical single or double cerclage sutures were removed at 37 weeks of gestation or whenever labor supervened.

\subsection{Randomization}

Blocked randomization using randomly drawn numbers was applied to assign the patients to receive one type of the two surgical modalities. The allocation order in randomization list was determined on consecutive patient registry order.

\subsection{Blinding}

The patients and statistician in this study were blind to the type of treatment.

\subsection{Endpoints}

Dichotomized gestational age at delivery was the main outcome of interest. Gestational age was dichotomized to form the efficacy variables as preterm labor $(\mathrm{GA}<37$ weeks) and extreme preterm labor (GA $<33$ weeks), the latter being the primary endpoint of this clinical trial. Gestational age $<37$ weeks, mean gestational age, neonatal mortality, 5-minute Apgar score, and PROM and neonatal hospital or intensive care unit stay were considered as secondary endpoints.

\subsection{Statistical Analysis}

Data were entered into the computer and analyzed using STATA 11 statistical software package. Based on data distribution, parametric and nonparametric statistics were used to compare the groups over continuous variable scales. Regarding the primary endpoint of interest, Chi-squared test followed by calculation of risk reduction (along with its 95\% confidence intervals) and number needed to treat (NNT) were performed or calculated. A pessimistic estimation of relative risk was made by replacing the zero value in a crosstabulation cell with an alternative as 1 . Statistical significance level was set at 0.05 .

\subsection{Ethical Issues}

The study was approved by committee of ethics in Urmia University of medical sciences. Based on ethical regulations of this committee, written informed consent was obtained from all the participants. They were assured of confidentiality of information and were informed about their freedom in participation or discontinuing it without any subsequent limitations due to their choice. 
TABLE 1: Age, gravid and gestational age before cerclage compared between trial groups.

\begin{tabular}{lccccc}
\hline Group & Statistic & Age & Gravid & Gestational age at cerclage time & Cervical length \\
\hline \multirow{3}{*}{ Traditional cerclage } & Mean & 26.3 & 2.4 & 15.5 & 25.3 \\
& Median & 24 & 2 & 15 & 23.5 \\
& SD & 7.6 & 1.3 & 2.1 & 5.8 \\
\hline \multirow{3}{*}{ Double celclage } & Mean & 27.3 & 3 & 16.1 & 24.5 \\
& Median & 28 & 3 & 15.5 & 23 \\
& SD & 5.9 & 1.4 & 2.2 & 4.6 \\
\hline \multirow{3}{*}{ Total } & Mean & 26.8 & 2.7 & 15.8 & 24.9 \\
& Median & 25 & 2.5 & 15 & 23 \\
& SD & 6.7 & 1.4 & 2.2 & 5.2 \\
\hline
\end{tabular}

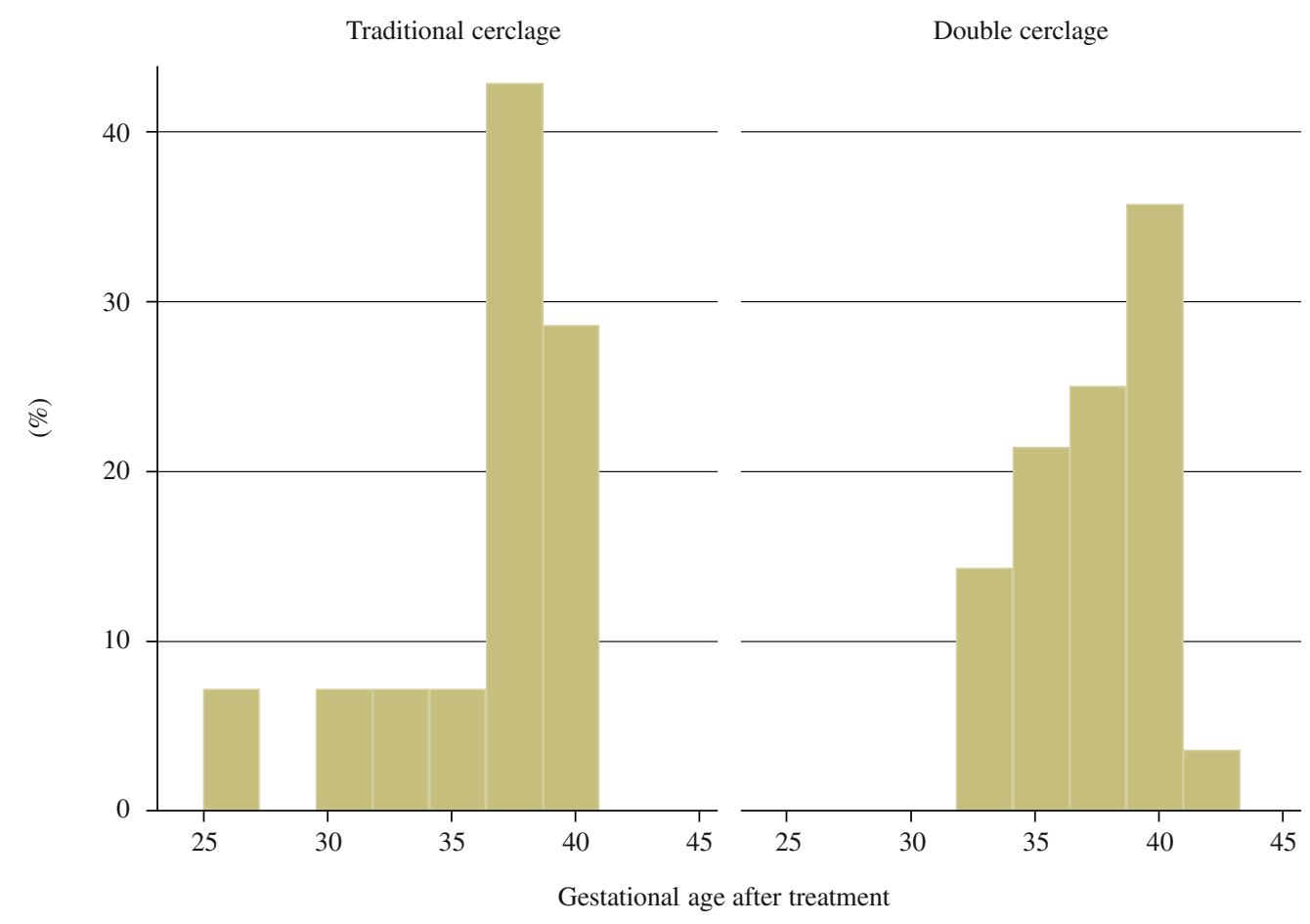

FIGURE 1: Histogram of gestational age after treatment with two treatment modalities.

\section{RESULTS}

Age, gestational age at cerclage time, and gravidity were not found to be statistically different between the groups. Descriptive statistics of these variables are given in Table 1. The rate of previous live births, abortions, and premature rupture of membranes was not found to be different between groups. Mean cervical length was measured to be $24.5 \mathrm{~mm}$ in double cerclage group being $0.8 \mathrm{~mm}$ shorter than in control group, but the difference was not statistically significant. Also the percentage of women with cervical length shorter than $25 \mathrm{~mm}$ was not statistically different between groups.

Mean gestational age compared between groups were 37.4 and 36.2 weeks, respectively, for investigational and control groups. The gestational age was 1.2 weeks longer for the group on double cerclage, but the difference was not found to be statistically significant using $t$-test. However, the distribution of gestational age appeared to be different (Figure 1). 
The rate of preterm birth before 37 weeks' gestation was similar for both groups. Preterm birth before 33 weeks of gestation was not experienced among any of the patients who received double cerclage, but five women in control group developed such an extreme preterm labor $(P<0.05)$. The absolute risk reduction in using double cerclage over traditional method was 18 percent (95\% confidence interval, $4 \%-32 \%)$. In this regard, the number needed to treat (NNT), that is, number of women to receive double cerclage in order to make one more woman benefit from this method, when compared with traditional cerclage, was calculated to be $5.6(P<0.05)$. Although due to zero frequency in one table cell it was not possible to calculate an accurate measure of relative risk, the minimum relative risk of preterm labor for traditional method was estimated by replacing the zero value with 1 as alternative to provide a conservative measure of relative risk that was then calculated to be equal to five. Premature rupture of membranes was observed in five double cerclage versus eight control group patients but the difference was not statistically different. Two neonatal deaths were observed during the first week after labor in control group but all live births after double cerclage continued alive. Mean 5-minute Apgar score was higher in double cerclage compared to traditional cerclage method $(P<0.05)$. Mean and median of 5-minute Apgar score were 8.96 and 9 for double cerclage, respectively, and the figures were 8.2 and 8 for control group. The neonatal ICU and hospitalization rate and length of stay measures were trivially better in double cerclage but without statistical significance.

\section{DISCUSSION}

As we may find in articles by McDonald and Shirodkar, cerclage during pregnancy is not a new term in reproductive literature possibly getting back to more than five decades ago $[3,5]$.

In this study we assessed the role of double cerclage in preventing preterm labor under 33 weeks of gestational age as we called it the "extreme preterm labor." Regardless of type of cerclage protocol, different GA cutoffs have been used in previous studies assessing the efficacy of cerclage.

Some studies have compared traditional cerclage methods with no treatment. Rust et al. aimed their study at comparing traditional cerclage with no cerclage in a randomized clinical trial on 113 patients concluding that the use of cerclage does not alter any perinatal outcome variable [9]. Another study found that therapeutic cerclage with bed rest reduced preterm delivery before 34 weeks of gestation and compound neonatal morbidity in women with risk factors and/or symptoms of cervical incompetence with a cervical length $<25 \mathrm{~mm}[10]$.

Similar to us To et al. considered the GA $<33$ weeks as the primary outcome of their study. They compared efficacy of Shirodkar cerclage with expectant management and they also found that insertion of a Shirodkar suture in women with a short cervix does not substantially reduce the risk of early preterm delivery [11]. Another study by Berhella et al. did not also find the cerclage to be more effective than bed rest to prevent preterm birth at $<35$ weeks of gestation [12]. Recently a multicenter randomized trial in the US found that in women with a prior spontaneous preterm birth GA $<34$ weeks and cervical length $<25$ $\mathrm{mm}$, cerclage reduced previable birth and perinatal mortality but did not prevent birth $<35$ weeks, unless cervical length was $<15 \mathrm{~mm}$ [4].

Woensdregt et al. may be the first ones to systematically compare single versus double stiches in preventing preterm labor. They found no measurable benefit for the placement of 2 stitches over 1 stitch during cervical cerclage in singleton pregnancies [13]. Similarl to us they did not find mean GA to be different, but with a low statistical power to reveal beneficence of double stitches. Contrary to us they did not find a significant difference in subcategories of GA also with low statistical power in most cases except when comparing GA $<28$ week. However, in present study double cerclage appeared to be better than traditional single cerclage. One major explanation for the different results of the study other than possible technical differences, may get back to the use of cohort study design by Woensdregt et al. The cohort design is more susceptible to confounding when compared with randomized clinical trial and trying to control the confound with multivariate analysis, in case of small studies and several confounders, results in low statistical power. However the idea behind double cerclage is not just providing a mechanically more stable barrier against expulsion pressure. The assumed plausibility mechanism is related to the role of mucus plug in preventing 
the ascending infection $[14,15]$. Hein et al. concluded that "The high immunoglobulin levels in combination with the presence of phagocytes suggest a potential for adaptive immune defense in the cervical mucus plug, which, together with innate immune factors, may act as an immunological gatekeeper protecting the fetomaternal unit against infection from the vagina" [15]. Although using double cerclage to preserve the mucus plug is somehow new in reproductive research, but the role of mucus plug preventing ascending infections gets back close to the time when traditional single cerclage was introduced $[16,17]$. Using a double cerclage procedure can be supported by two plausible mechanisms; one being double strengthened weak cervix, and the other can be preventing ascending infection by preserving the mucus plug [18]. A recent Taiwanese randomized trial has also compared double cerclage done on 17 pregnant women having incompetent cervix, with traditional cerclage on 34 other women in similar situation. They found the double cerclage to be more effective than traditional cerclage in increasing the gestational age or decreasing the preterm labor rate. Similarly with us, they also found just better descriptive statistics regarding measures related with neonatal health [19]. Although our study found statistically significant difference regarding Apgar score, but generally it seems that both studies are underpowered to assess neonatal outcomes.

Like most other clinical trials we also did not perform any test to assess the incidence and presence of infection. This should be considered as a limitation. Although the confounding role of infection can be controlled through randomization, assessing the infection rate can at least help to investigate differentiation of the two possible mechanisms for the higher efficacy of double cerclage as discussed above.

\section{CONCLUSION}

Double cerclage appeared to have higher efficacy than traditional cerclage in preventing preterm labor $<33$ weeks of gestation. Larger-scale studies are needed to assess the efficacy on neonatal outcomes and to identify possible target pregnancies for higher efficacy of double cerclage over traditional cerclage.

\section{REFERENCES}

[1] S. G. Gabbe, J. R. Niebyl, and J. L. Simpson, Obstetrics: Normal and Problem Pregnancies, Churchill Livingstone, 2007.

[2] A. H. Ansari and R. A. Reynolds, "Cervical incompetence: a review," Journal of Reproductive Medicine, vol. 32, no. 3, pp. 161-171, 1987.

[3] V. N. Shirodkar, "A new method of operative treatment for habitual abortion in the second trimester of pregnancy," Antiseptic, vol. 52, pp. 299-300, 1955.

[4] J. Owen, G. Hankins, J. D. Iams et al., "Multicenter randomized trial of cerclage for preterm birth prevention in high-risk women with shortened midtrimester cervical length," American Journal of Obstetrics and Gynecology, vol. 201, no. 4, pp. 375.e1-375.e8, 2009.

[5] I. A. McDonald, "Suture of the cervix for inevitable miscarriage," Journal of Obstetrics and Gynaecology of the British Empire, vol. 64, pp. 346-350, 1957.

[6] J. M. Svigos, J. M. Dodd, and J. S. Robinson, "Threatened and actual preterm labor including mode of delivery," in High Risk Pregnancy: Management Options, pp. 1075-1090, Elsevier Saunders, 4th edition, 2011.

[7] M. Noori, R. B. Helmig, M. Hein, and P. J. Steer, "Could a cervical occlusion suture be effective at improving perinatal outcome?" BJOG, vol. 114, no. 5, pp. 532-536, 2007.

[8] H. Sadeghi Bazargani and S. Hajebrahimi, "Evidence-based urology: how does a randomized clinical trial achieve its designed goals?" Urology Journal, vol. 8, pp. 88-96, 2011.

[9] O. A. Rust, R. O. Atlas, J. Reed, J. Van Gaalen, and J. Balducci, "Revisiting the short cervix detected by transvaginal ultrasound in the second trimester: why cerclage therapy may not help," American Journal of Obstetrics and Gynecology, vol. 185, no. 5, pp. 1098-1105, 2001.

[10] S. M. Althuisius, G. A. Dekker, P. Hummel, D. J. Bekedam, and H. P. Van Geijn, "Final results of the cervical incompetence prevention randomized cerclage trial (CIPRACT): therapeutic cerclage with bed rest versus bed rest alone," American Journal of Obstetrics and Gynecology, vol. 185, no. 5, pp. 1106-1112, 2001. 
[11] M. S. To, Z. Alfirevic, V. C. F. Heath et al., "Cervical cerclage for prevention of preterm delivery in women with short cervix: randomised controlled trial," Lancet, vol. 363, no. 9424, pp. 1849-1853, 2004.

[12] V. Berghella, A. O. Odibo, and J. E. Tolosa, "Cerclage for prevention of preterm birth in women with a short cervix found on transvaginal ultrasound examination: a randomized trial," American Journal of Obstetrics and Gynecology, vol. 191, no. 4, pp. 1311-1317, 2004.

[13] K. Woensdregt, E. R. Norwitz, M. Cackovic, M. J. Paidas, and J. L. Illuzzi, "Effect of 2 stitches vs 1 stitch on the prevention of preterm birth in women with singleton pregnancies who undergo cervical cerclage," American Journal of Obstetrics and Gynecology, vol. 198, no. 4, pp. 396.e1-396.e7, 2008.

[14] M. Hein, E. V. Valore, R. B. Helmig, N. Uldbjerg, and T. Ganz, "Antimicrobial factors in the cervical mucus plug," American Journal of Obstetrics and Gynecology, vol. 187, no. 1, pp. 137-144, 2002.

[15] M. Hein, A. C. Petersen, R. B. Helmig, N. Uldbjerg, and J. Reinholdt, "Immunoglobulin levels and phagocytes in the cervical mucus plug at term of pregnancy," Acta Obstetricia et Gynecologica Scandinavica, vol. 84, no. 8, pp. 734-742, 2005.

[16] R. Rozansky, S. Persky, and B. Bercovice, "Antibacterial action of human cervical mucus," Proceedings of the Society for Experimental Biology and Medicine, vol. 110, pp. 876-881, 1962.

[17] H. Zuckerman, A. Kahana, and S. Carmel, "Antibacterial activity of human cervical mucus," Gynecologic Investigation, vol. 6, no. 5, pp. 265-271, 1975.

[18] N. J. Secher, C. D. McCormack, T. Weber, M. Hein, and R. B. Helmig, "Cervical occlusion in women with cervical insufficiency: protocol for a randomised, controlled trial with cerclage, with and without cervical occlusion," BJOG, vol. 114, no. 5, pp. 649.e1-649.e6, 2007.

[19] Y. L. Tsai, Y. H. Lin, K. M. Chong, L. W. Huang, J. L. Hwang, and K. M. Seow, "Effectiveness of double cervical cerclage in women with at least one previous pregnancy loss in the second trimester: a randomized controlled trial," Journal of Obstetrics and Gynaecology Research, vol. 35, no. 4, pp. 666-671, 2009.

\section{This article should be cited as follows:}

Farzaneh Broumand, Fatemeh Bahadori, Tahereh Behrouzilak, Zahra Yekta, and Farkhondeh Ashrafi, "Viable Extreme Preterm Birth and Some Neonatal Outcomes in Double Cerclage versus Traditional Cerclage: A Randomized Clinical Trial," TheScientificWorldJOURNAL, vol. 11, pp. 1660-1666, 2011. 


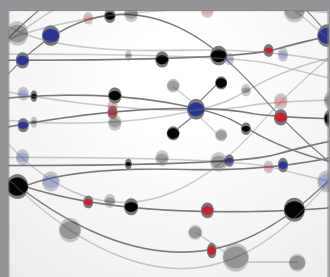

The Scientific World Journal
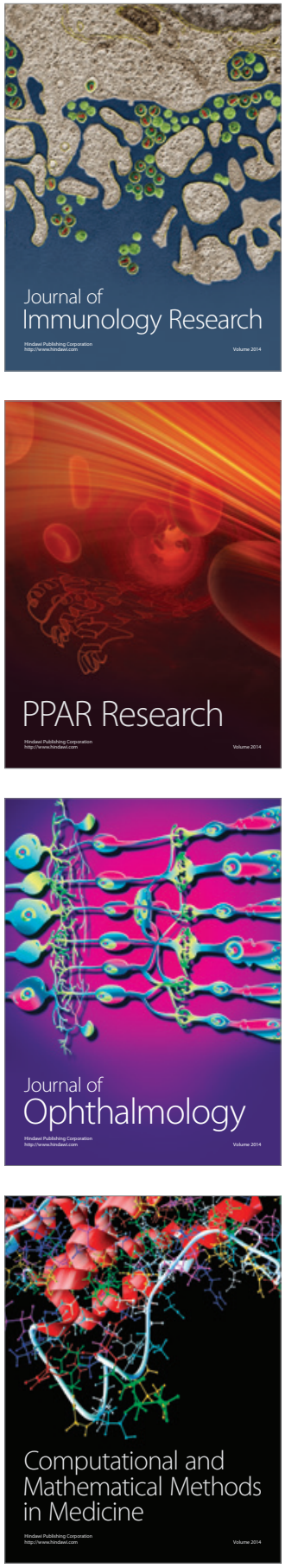

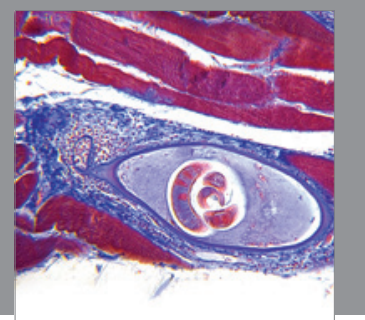

Gastroenterology

Research and Practice
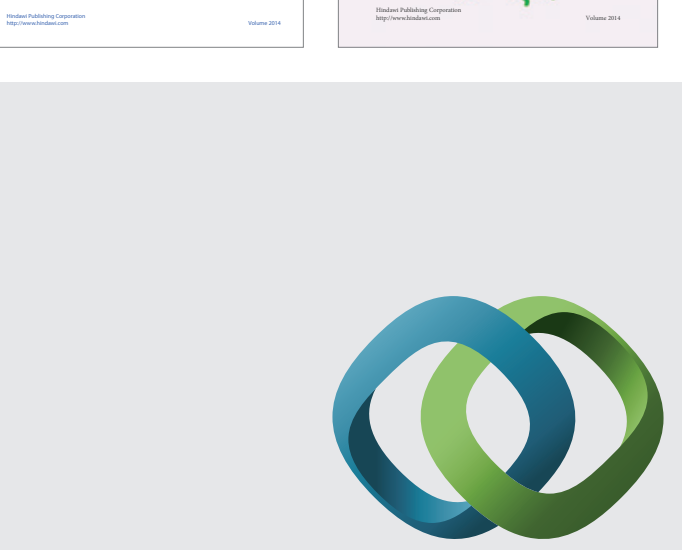

\section{Hindawi}

Submit your manuscripts at

http://www.hindawi.com
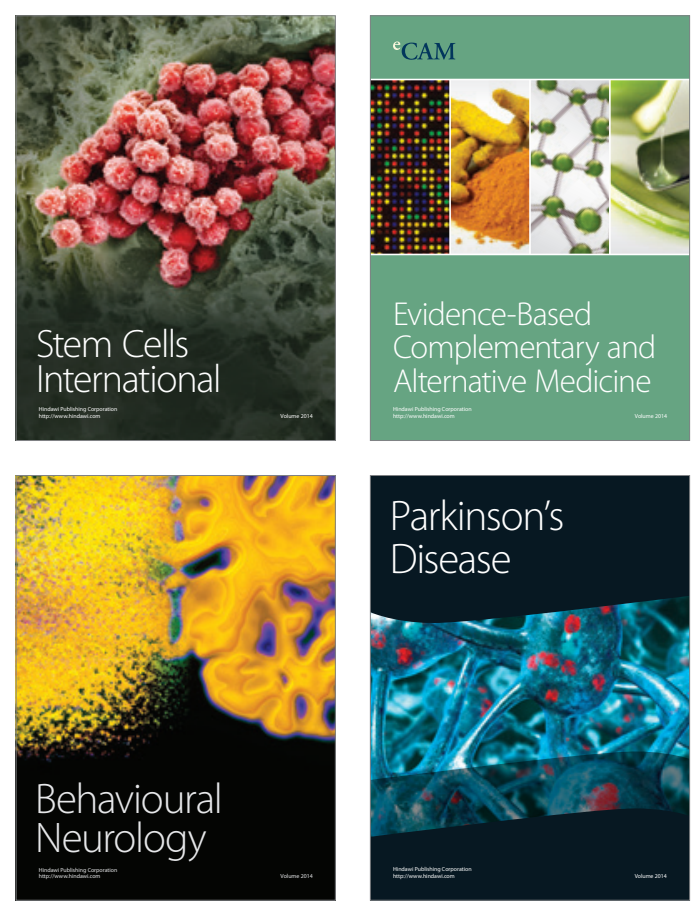

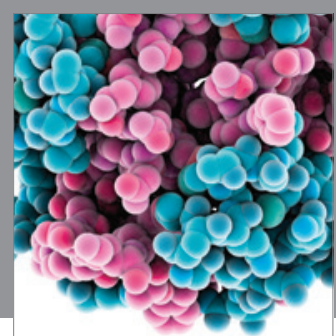

Journal of
Diabetes Research

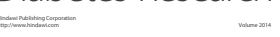

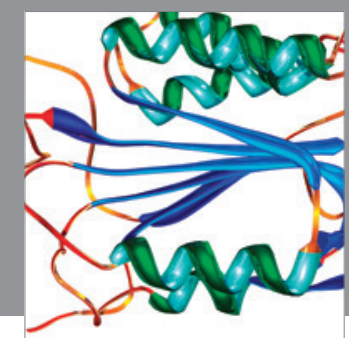

Disease Markers
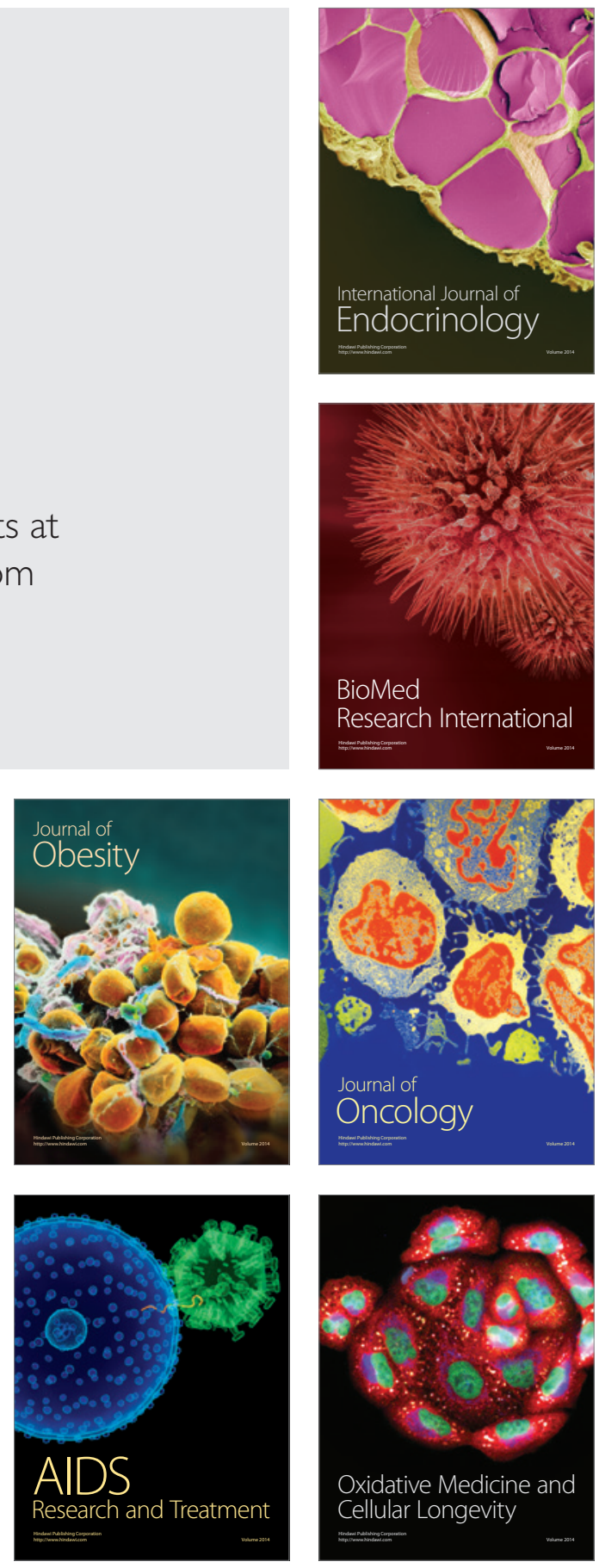